\title{
Léon Gérin (1863-1951)
}

(1931)

\section{"La famille canadienne-française, sa force, ses faiblesses. Le paysan de Saint-Irénée, hier et aujourd'hui."}

\author{
Un document produit en version numérique par Jean-Marie Tremblay, bénévole, \\ professeur de sociologie au Cégep de Chicoutimi \\ Courriel: jimt sociologue@,videotron.ca \\ Site web: http://pages.infinit.net/sociojmt
}

Dans le cadre de la collection: "Les classiques des sciences sociales"

Site web: http://www.uqac.uquebec.ca/zone30/Classiques_des_sciences_sociales/index.html

Une collection développée en collaboration avec la Bibliothèque

Paul-Émile-Boulet de l'Université du Québec à Chicoutimi

Site web: http://bibliotheque.uqac.uquebec.ca/index.htm 
Cette édition électronique a été réalisée par Jean-Marie Tremblay, bénévole, professeur de sociologie au Cégep de Chicoutimi à partir de :

"La famille canadienne-française, sa force, ses faiblesses. Le paysan de Saint-Irénée, hier et aujourd'hui." (1931)".

Un article publié originalement dans la Revue trimestrielle canadienne, $\mathrm{n}^{\circ}$ 19, mars 1932, pp. 35-63. Texte d'une conférence prononcée le 24 octobre 1931, à l'Institut pédagogique de Montréal. Un article reproduit dans $L a$ société canadienne-française. (pp. 45-67) Études choisies et présentées par Marcel Rioux et Yves Martin. Montréal: Editions Hurtubise HMH, ltée, 1971, 404 pp.

Polices de caractères utilisée :

Pour le texte: Times, 12 points.

Pour les citations : Times 10 points.

Pour les notes de bas de page : Times, 10 points.

Édition électronique réalisée avec le traitement de textes Microsoft Word 2001 pour Macintosh.

Mise en page sur papier format

LETTRE (US letter), 8.5' x 11'”)

Édition complétée le 9 juillet 2004 à Chicoutimi, Québec.

\section{Fait avec}

Macintosh 


\title{
Table des matières
}

\author{
Introduction
}
A. Le groupement familial quasi-communautaire
B. La famille communautaire en train de s'émanciper
C. La petite culture familiale, vivrière, traditionnelle
D. Le double appui de la culture paysanne: simple récolte des productions spontanées, industries domestiques
E. La disparition graduelle des productions spontanées
F. La renaissance des arts domestiques
G. L'habitant dans les mines et les transports
H. Fonction éducatrice de la famille
I. La double réaction du type familial sur la société canadienne
J. Action du commerce lointaine et pourtant dissolvante
K. Rôle effacé de la culture intellectuelle et des Professions libérales
L. Les deux corporations dirigeantes 


\section{Introduction}

Retour à la table des matières

Dans une première communication, le 10 octobre dernier, je vous indiquais un fait dont il faut d'abord tenir compte dans toute étude du milieu social : sa décomposition spontanée, pour qui l'observe de près, en groupements organiques, c'est-à-dire en organes répondant à des besoins essentiels, permanents de notre vie en société. Ces groupements organiques, vous disaisje alors, se répartissent en douze ou quinze classes, dont les plus fondamentales sont la famille et l'atelier de travail.

Et j'ajoutais : tout groupement organique se résout en six éléments constitutifs, qui sont le personnel humain qui le compose; la fonction (ou les fonctions) qu'il remplit; les moyens d'existence dont il dispose en vue de remplir plus ou moins efficacement son rôle social ; le mode d'existence qui en résulte pour les membres du groupe; les phases d'existence ou d'évolution par lesquelles est passé le groupement et qui ont influé sur sa manière d'être ou d'agir; enfin, ses rapports divers avec les autres groupements organiques qui lui sont associés ou superposés; en d'autres termes, sa situation, son importance relative dans le complexus de la vie sociale.

De prime abord, tout cela a dû vous paraître bien sommaire, bien abstrait, et sans doute ne s'est représenté que de manière vague à votre esprit. Cependant, le groupement social organique, par tous ses éléments constitutifs, tombe sous les sens, et se trouve dès lors parfaitement observable dans des conditions propices à l'application des procédés ordinaires de la connaissance : l'analyse, la comparaison, la classification. 
Aussi, en vue de vous aider à débrouiller la confusion que présente à l'observateur novice le milieu social même le plus simple, vous ai-je suggéré deux procédés pratiques d'emploi relativement facile par toute personne douée d'esprit de suite et de volonté : 1) la monographie de famille ouvrière, échantillonnage en couche profonde; s'aidant à l'occasion de 2) l'enquête sociale, vue d'ensemble, plus rapide, plus embrassante, mais aussi plus superficielle et qui ne saurait se passer entièrement du recours à la monographie.

Vous m'invitez maintenant, - et c'est beaucoup d'honneur, - à vous parler de la famille canadienne-française, de ses éléments de force ou de faiblesse ; et cela me fournit une excellente occasion de faire sous vos yeux l'essai des principes posés dans ma première conférence, de mettre à l'épreuve la méthode d'observation dont je vous ai proposé l'emploi. Car si ces principes sont justes, si cette méthode d'observation monographique est féconde, l'étude concrète d'une famille ouvrière, surtout dans un pays comme le Canada français, resté le château fort pour ainsi dire de la famille, devrait éclairer d'un jour très vif toute notre organisation sociale ; à condition, toutefois, que l'échantillon ait été judicieusement prélevé, de manière à être représentatif du type le plus général.

Comment procéderons-nous au choix préalable d'un échantillon de la famille canadienne ? Première condition, cette famille devra habiter la campagne ; car le Canadien français est foncièrement campagnard. Deuxième condition, complémentaire de la précédente, le milieu rural où nous nous mettrons en observation ne devra avoir été que fort peu atteint par les influences émanant des centres urbains, par le contact avec le mouvement industriel et commercial. Troisième desideratum, qui n'est pas toujours facile de réalisation : les observations devront s'étendre sur un nombre d'années suffisant pour permettre l'analyse de la famille non seulement sous l'aspect statique, à l'état de repos, mais sous l'aspect dynamique, c'est-à-dire dans les péripéties de son existence, en train d'adaptation ou d'évolution.

À ces divers points de vue, je n'en connais pas qui réponde mieux aux exigences, ou dont l'étude soit plus suggestive que la famille rurale de SaintIrénée, côte nord du Saint-Laurent, en aval de Québec, observée et décrite dès 1861 et 1862 par un adepte de LePlay, et dont à deux reprises, en 1920 et 1929, il m'a été donné de compléter et mettre à jour l'esquisse monographique.

Dans ce qui forme aujourd'hui le comté de Charlevoix, à 82 milles en bas de Québec, et dominant le fleuve, large ici comme un bras de mer, se campe au flanc du plateau laurentien, dans un horizon grandiose, la pittoresque campagne de Saint-Irénée. Par un beau jour de l'été de 1862, deux hommes grimpaient la pente raide et sablonneuse qui, du presbytère, sis à mi-côte, conduit au plateau. 
L'un d'eux était prêtre, l'abbé Jean Mailley, fils d'un officier de garnison de Besançon, dans l'ancienne Franche-Comté, mais depuis deux ans exerçant les fonctions curiales dans cette paroisse canadienne du bas du fleuve. Son compagnon de route était un compatriote, M. Gauldrée-Boilleau, alors consul de France à Québec et qui plus tard, transféré à New-York et devenu baron du second Empire, devait disparaître de manière tragique dans la tourmente qui précipita la chute de Napoléon III.

Ils allaient rendre visite à un brave paroissien du curé Mailley, Isidore Gauthier, cultivateur, dont la solide maison de bois, mise en peinture blanche, avec toit et contrevents colorés en rouge, couronnait la haute falaise caressée par le vent piquant du nord-est et d'où le regard s'étend à perte de vue sur la nappe bleuâtre, tantôt immobile et tantôt moutonnante, de l'immense SaintLaurent.

\section{A. Le groupement familial quasi-communautaire}

\section{$\underline{\text { Retour à la table des matières }}$}

La famille d'Isidore Gauthier comprenait à ce moment neuf personnes le père, âgé de quarante ans ; sa femme, Sarah Girard, trente-neuf ans; et sept enfants : Marie-Rose, la fille aînée, âgée de dix-huit ans, et depuis un an mariée à un villageois ; Marie-Hortense, âgée de quinze ans, au foyer paternel ; Marie-Césarine, âgée de quatorze ans; Marie-Démerise, âgée de douze ans ; Isidore Gauthier, premier fils, âgé de dix ans; Sarah, âgée de six ans; joseph, âgé de quatre ans.

Gauldrée-Boilleau, qui a recueilli sur place les matériaux de cette monographie, avec la collaboration très active du curé, son ami, affirme que les familles de huit ou dix ou même de douze enfants sont nombreuses à Saint-Irénée. Elles n'égalent pourtant pas la fécondité des familles de pêcheurs de la péninsule de Gaspé, où les productions spontanées de la mer tiennent une place encore plus large dans les ressources de l'habitant. Là les familles de dix-huit ou vingt enfants, à ce qu'on lui a assuré, s'observent assez fréquemment. 
La population de Saint-Irénée, en 1861, était de 1,060 âmes et se répartissait entre 100 familles, soit une moyenne de 10 ou 11 personnes par famille. L'adoption d'orphelins se pratique couramment; et les parents ou voisins qui ont recueilli ces enfants les traitent comme s'ils étaient leurs propres enfants. Beaucoup d'enfants d'émigrants irlandais, décimés par le typhus, ont été ainsi incorporés à des familles de la région.

Quant à Isidore Gauthier, dont la famille fait le sujet de la monographie du Paysan de Saint-Irénée, mise en oeuvre sous la signature de GauldréeBoilleau, il était l'aîné d'une famille de cinq enfants, tous du sexe masculin. La moitié du terrain appartenant à la fabrique et à l'église paroissiale de SaintIrénée provient d'un don du père et de la mère d'Isidore. Celui-ci a hérité de la maison paternelle et des champs qui l'entouraient. Les deux frères d'Isidore, plus jeunes, ont reçu chacun une terre d'une valeur à peu près équivalente ; mais à la condition de se joindre à lui pour établir les deux derniers dès qu'ils auraient atteint l'âge de vingt et un ans; et que chacun serait mis alors en possession d'une terre de 2,000 francs, d'un cheval, d'une vache, d'un bœuf et de quelques moutons.

Gauldrée-Boilleau constate encore que «dans certaines paroisses, c'est presque toujours le plus jeune qui hérite de la propriété paternelle. On se marie jeune au Canada, et les mariages y sont féconds. Quand l'aîné des garçons arrive à l'âge de s'établir, ses père et mère sont encore en possession de toutes leurs forces et ne songent même pas à renoncer à travailler ; ils se contentent donc de fournir à leur fils les moyens d'ouvrir une exploitation agricole dans une paroisse peu distante de celle où ils résident, mais moins anciennement habitée et dans laquelle, par conséquent, les terres sont à meilleur marché. Le même plan est adopté à l'égard du second garçon, du troisième et ainsi de suite ; quand le dernier de tous est en état de diriger une propriété rurale, le père approche de la vieillesse et sent le besoin de se reposer ; si le plus jeune est intelligent, il devient maître du domaine, moyennant une pension viagère assurée à ses parents par contrat passé devant notaire. »

En disciple averti de Frédéric LePlay, Gauldrée-Boilleau insiste sur cette pratique de la transmission intégrale dans le pays de Québec :

«Il n'est point d'usage de morceler la propriété foncière comme cela se pratique en France. Le chef de famille s'applique à réaliser des économies et acquiert une terre pour chacun de ses fils en âge de la cultiver. Si ses moyens ne lui permettent pas d'accomplir entièrement cette tâche, à laquelle il attache une importance extrême, il lègue le bien patrimonial au plus intelligent de ses garçons, en lui imposant la charge d'aider ses frères et sœurs et de les établir, petit à petit, d'une manière convenable. »

Puis, après avoir décrit sommairement le régime du travail, les procédés de culture et d'élevage, les industries domestiques exercées par les divers 
membres de la famille ; après avoir indiqué en grand détail les biens mobiliers ou immobiliers dont elle est propriétaire, la composition du troupeau, du matériel agricole; fait l'inventaire de son mobilier meublant, de son linge de ménage; énuméré les divers produits qu'elle récolte et dont la presque totalité est utilisée ou consommée à la maison, Gauldrée-Boilleau conclut en ces termes significatifs et plutôt admiratifs :

«Le domaine patrimonial reste intact au milieu des vicissitudes que subit la famille, qui se divise sans que lui-même soit morcelé ; c'est le foyer d'où rayonnent les émigrations successives qui vont peupler les paroisses voisines, et le centre traditionnel où, à plusieurs générations de distance, les personnes qui descendent d'une souche commune sont heureuses de se rencontrer ».

Ainsi, dans ce coin perdu de notre Canada français, le fonctionnaire d'un gouvernement imbu de tendances conservatrices se persuadait volontiers qu'il avait bel et bien sous les yeux un exemplaire nettement caractérisé de cette fameuse famille souche que LePlay avait signalée à la France et à l'Europe comme le prototype de la bonne organisation de la vie domestique; bien supérieure à la famille patriarcale ou communautaire de l'Orient ; bien supérieure surtout à la famille ébranlée ou instable de l'Europe occidentale.

Entre-temps, les adeptes les plus perspicaces et les plus consciencieux de LePlay se sont aperçus que sous cette appellation de famille souche, le maître et ses premiers collaborateurs avaient confondu deux types divergents, opposés : la famille particulariste, à fort développement de l'initiative personnelle, qui distingue au premier chef les peuples de l'Europe septentrionale ; et la fausse famille souche, observable dans les pays de l'Occident de l'Europe, et qui n'est en somme qu'une variété, ou atténuation du type ancien de la famille patriarcale ou communautaire. Mais passons.

\section{B. La famille communautaire en train de s'émanciper}

$\underline{\text { Retour à la table des matières }}$

En septembre 1920, presque soixante ans après cette mémorable visite du curé Maillay et de Gauldrée-Boilleau chez Isidore Gauthier, votre humble serviteur, parti de Québec quelques heures auparavant, arrivait par le bateau à Saint-Irénée ; et, sur le quai, au clair d'une froide lune d'automne, la conversation suivante s'engageait: 
Un Saint-Irénéen: Vous cherchez un hôtel ? - Moi. Je cherche plutôt un certain M. Gauthier.

- Lui : Mon nom est Gauthier, Pitre Gauthier, hôtelier, pour vous servir.

- Moi: C'est Isidore Gauthier que je cherche.

- Lui : Isidore ? Il y a belle lurette qu'il n'est plus de ce monde.

- Moi : Isidore père, je vous crois volontiers; s'il vivait encore, il serait presque centenaire. Mais il avait un fils du même nom qui aurait à peine soixante-dix ans.

- Lui : Vous voulez dire Isidore fils, celui que tout le monde appelait «Gauthier », tout court ? Eh bien, celui-là aussi est mort, tout comme son père. - Moi : Mais Isidore Gauthier fils a dû laisser des descendants, j'aimerais causer avec eux.

- Lui : Toute cette famille de Gauthier a quitté la paroisse il y a longtemps déjà. - Moi : J'en suis fâché ; mais enfin, la terre, la maison, les dépendances sont encore là. Voudrez-vous m'y conduire ?

- Lui : Vous ne savez donc pas ? La terre a été vendue, la maison débâtie, les matériaux transportés au loin, utilisés pour d'autres constructions. Il ne reste que les fondations de ce qui était sa maison.

J'étais confondu. Ainsi s'écroulait piteusement la glorieuse vision un instant aperçue d'une famille souche canadienne sur le modèle classique de celle de LePlay. « Mais quelle est donc la catastrophe, m'écriai-je, quel est le revers de fortune qui a terrassé ce fort d'entre les forts, qui l'a déraciné du sol natal, lui et les siens ?» Mon interlocuteur me regardait d'un air surpris. «Mais, monsieur, finit-il par dire, il n'y a pas eu de revers de fortune. Isidore n'était pas en mauvaises affaires ; son unique mobile en s'éloignant d'ici était l'espoir d'améliorer ses propres conditions d'existence, et d'assurer l'établissement futur de ses enfants. Il s'est fixé dans la vallée du Saguenay, et je vous assure que ses descendants y ont grandement prospéré. »

Dès le lendemain, mon hôtelier, guide précieux, parfaitement renseigné sur les gens et les choses du pays, me conduisait chez d'autres Gauthier, de la parenté d'Isidore, presque ses sosies. Tirés par un petit cheval canadien, nous grimpions la longue côte sablonneuse, nous jetions en passant un coup d'œil sur l'emplacement désert des bâtisses d'Isidore, nous roulions tranquillement sur la crête de cette « contrée extrêmement pittoresque », de ce pays sillonné de torrents, encadré par la chaîne des Laurentides. Tableau imposant mais froid et sévère. 
Nous avions devant nous, remplissant l'horizon du nord, une mer de sommets ondulants et de vallons aux contours indécis.

Soudain, le long boyau de la route de sable jaune s'infléchit vers l'ouest ; nous franchissons une gorge étroite au fond de laquelle murmure le modeste affluent du ruisseau jean-Noël, et nous voici arrivés devant la maison longue et basse de Méderile Gauthier. Celui-ci est un petit-neveu d'Isidore père ; beau type d'homme, taille élevée, figure intelligente, démarche énergique, il habite ici avec sa mère, sa femme et plusieurs enfants, dont un est marié au foyer et un autre est installé à proximité sur une terre qu'il met en valeur.

Or tout cela, dans sa simplicité, dans sa sobriété, ne manque pas d'une certaine poésie et comporte un enseignement. Poésie de la grande nature septentrionale du bas Saint-Laurent ; froide, impassible, mais impressionnante aussi; berceau d'une race vigoureuse, inexpugnable. Poésie, et leçon aussi, de l'ancêtre paysan fondateur délaissé d'une nationalité nouvelle, conservateur obstiné de ce qu'il y avait de bon et de fort dans la tradition médiévale française.

Quel bon accueil on nous fait sous ce toit hospitalier! Quand le maître de céans apprend que je suis venu à Saint-Irénée pour me renseigner sur la manière de vivre des habitants, il m'ouvre la porte de sa maison ; il m'invite à m'installer chez lui, ce qui me dispenserait du long trajet à faire soir et matin entre sa terre du plateau et l'hôtel du village bordant le fleuve. La mère de mon hôte, vénérable matrone, âgée de soixante-dix-neuf ans, se montre particulièrement accueillante.

C'est une Harvey, originaire de l'île aux Coudres, proche de Saint-Irénée. Anicet Gauthier, père de Méderile, l'avait épousée lorsqu'elle était encore jeune institutrice. Ce nom de Harvey atteste bien sa descendance écossaise. En effet les highlanders formaient un contingent important de l'armée d'invasion de Wolfe. Après la conquête, ils s'établirent en nombre dans les seigneuries concédées dans le bas du fleuve à leurs officiers, les Nairne, les Fraser, etc. Communautaires autant et plus que les Canadiens, ils sympathisèrent avec eux, s'unirent à eux par des mariages et ne tardèrent pas à s'assimiler et se fondre dans le milieu rural québécois.

«Ah qu'on aime donc à retrouver les siens ! » dit en me touchant l'épaule, cette bonne vieille, qui me rencontre pour la première fois. Et avec le cœur ouvert, l'esprit primesautier, la parole abondante de l'ancêtre celtique, elle m'entretient sur le sujet du livre de l'ancien curé Mailley (prononcé Mailly) dont elle connaît toute l'histoire. "Je suis l'aïeule », déclara-t-elle avec émotion, «mais la bisaïeule est là-haut. » Elle montrait du doigt le ciel. 
Hélas ! lorsque je revins à Saint-Irénée, neuf ans plus tard, l'aïeule était allée rejoindre là-haut la bisaïeule.

On le voit, nous nageons ici en pleine tradition communautaire. Sur ce foyer canadien plane encore la grande ombre de l'ancêtre au nom biblique : Abraham ou Brahm. Gauthier, qui a laissé dans le pays le souvenir d'un patriarche, et qui fut l'initiateur de son groupe aux procédés de l'exploitation agricole. Notamment, il aurait été, s'il faut en croire la tradition, le premier dans la région, sinon dans la province, à se munir d'une moissonneuse.

Mais en voilà assez pour le moment sur le personnel de la famille et son groupement au foyer. Sortons de la maison et rendons-nous compte de l'organisation de l'atelier de travail. Car, à l'image de toute famille rurale du Canada français, celle de Méderile Gauthier n'est pas un simple agrégat de parents : c'est aussi un groupement de travailleurs agricoles. Famille et atelier rural sont intimement unis et réagissent fortement l'un sur l'autre, nous allons le voir.

\section{La petite culture familiale, vivrière, traditionnelle}

$\underline{\text { Retour à la table des matières }}$

L'exploitation agricole est restreinte dans les limites étroites des besoins modestes et immédiats de la famille et de ses ressources en main-d'œuvre. Les cultures sont diversifiées; elles comprennent plusieurs sortes de légumes; pommes de terre, gourganes (c'est la fève des marais), fèves à café (c'est la fèverole), etc., mais toutes sur de faibles étendues. Les céréales occupant plus de place, le blé par exemple, et surtout l'avoine. Dans l'assolement biennal, généralement suivi, elles alternent avec le pâturage ou le friche, et moins souvent, avec le foin. De même, le troupeau comprend des chevaux, des bêtes à cornes, des moutons, des porcs et des volailles, mais en nombre restreint, si l'on tient compte de la superficie exploitée. Le progrès réalisé sous ce rapport depuis l'époque du vieil Isidore n'est guère marqué.

Les procédés de culture ne sont nullement intensifs. Ils se calquent sur la productivité naturelle du lieu et restent toujours dans son étroite dépendance. Ainsi les hautes terres de Saint-Irénée à cause de la texture peu serrée de leur sol, qui laisse facilement filtrer les eaux, sont de manière générale propices à la culture des céréales, surtout dans les étés de pluie abondante. D'autre part, elles se prêtent beaucoup moins bien à la création de prairies artificielles 
durables. Les champs de trèfle et de graminées sont ici rares et de faible étendue.

L'assolement généralement suivi est biennal ou, plus exactement, doublebiennal : soit, deux récoltes successives de grains alternant avec le friche, la jachère nue, ou, plus rarement, la jachère herbée. Par suite de l'extrême réduction du troupeau, la dépense d'engrais est restreinte au minimum. Rien ne saurait mieux caractériser un système d'exploitation reposant en très grande partie sur la simple récolte, tirant du fond tout ce que recèle sa fertilité première et spontanée.

Tout cela est marqué au coin de la plus ancienne tradition. Dès ma première visite, en 1920, j'avais été vivement frappé de l'apparence d'ancienneté et de vétusté de l'installation et de l'outillage chez Méderile Gauthier. Comme la maison d'habitation, les bâtiments de ferme étaient assez spacieux et disaient l'aisance du propriétaire ; longs et de faible hauteur, ils ne manquaient pas d'un certain pittoresque, mais semblaient ployer sous le faix de leur épaisse couverture de chaume.

« Nous continuons à couvrir en chaume », m'avait expliqué mon hôte, « car la paille ne nous coûte rien, et si nous y renoncions, il nous faudrait la remplacer par du bois, que nous payerions aujourd'hui fort cher. » L'outillage de ferme me parut aussi fort ancien, par exemple ce manège à plan horizontal et arbre vertical servant pour les battages, et qui a presque partout, de longue date, cédé la place aux trépigneuses à plan incliné.

Et cet assolement biennal tenu en honneur ici, que pratiquait Isidore Gauthier père, il y a soixante-dix ans, lorsque Gauldrée-Boilleau mettait en oeuvre sa monographie du paysan canadien, cet assolement a été suivi sur les bords du Saint-Laurent durant tout le régime français, et de manière exclusive jusque vers la fin du siècle dernier. Il survit même de nos jours dans plus d'une région de la province. Or sait-on que, à travers le Moyen Age, il nous est venu des Romains?

On conçoit que dans un milieu traditionnel, isolé comme celui-ci, le progrès des méthodes soit lent à se produire. Cependant, grâce à l'initiative des plus entreprenants, il finit par se faire jour.

Il y a plusieurs années déjà, dès avant ma première visite à Saint-Irénée, Méderile Gauthier s'était associé avec quelques voisins pour capter l'eau de sources éloignées, dont le trop-plein par le moyen de conduites d'eau fut amené jusqu'aux bâtiments des divers intéressés, laissant ainsi disponibles des bras qui précédemment dépensaient leur force à charrier l'eau servant à abreuver les bêtes à l'étable. 
Lors de ma deuxième visite à Saint-Irénée, en 1929, je constatai, non sans éprouver un certain regret de folkloriste, que la vieille masure pseudo-normande de Méderile Gauthier avait été démolie - il ne restait plus qu'une partie du fournil avec sa cheminée, - la longue grange-étable avait été démolie elle aussi ; l'une et l'autre remplacées par des constructions d'aspect plus banal, mais offrant, à ce que m'assurait le propriétaire, plus de logement et de confort.

Chez Clovis Gauthier, - cultivateur de ce même voisinage que nous avions eu la bonne fortune de rencontrer la veille dans le train qui nous amenait de Québec, - nous faisons la même constatation que chez Méderile, du moins en ce qui regarde l'habitation. La masure antique, mal éclairée, difficile à tenir chaude par les grands froids d'hiver, a été remplacée par une maison à deux étages, de style plus moderne. D'autre part, le système général d'exploitation, reste à peu près le même : culture répétée d'une céréale sur le même terrain, suivie d'autant d'années de pacage, avec complément de vastes étendues qui restent en friche.

Clovis m'explique que cette avoine luxuriante, au long chaume, robuste aussi, puisque en septembre encore elle est debout, ayant jusque-là résisté à la verse, il l'a emblavée sur des champs où ses vaches avaient pâturé deux années de suite. Il faut dire que, cet été de 1929, assez pluvieux, a été particulièrement favorable aux cultures en terres hautes et sableuses comme celles de Saint-Irénée. Nulle part le long de la route, pas même sur les sols d'alluvion de Château-Richer ou de Saint-Joachim (Côte de Beaupré), je n'ai observé de plus beau grain qu'il ne s'en trouve ici.

Par ses traits essentiels, l'exploitation de Clovis Gauthier ne diffère pas sensiblement de celle de ses voisins les plus entendus. À perte de vue dans la direction du nord, se déroulent des friches ou pâturages permanents, embroussaillés par endroits. Peu de prairies artificielles ; la paille des céréales utilisée non seulement comme litière à l'étable, mais comme partie importante ou principale de la ration d'hivernement des bêtes à cornes. Pour éviter la verse, Clovis coupe son avoine encore «verdaude », c'est-à-dire sans attendre la complète maturité du grain. Troupeau fort réduit; cultures diversifiées, mais sur de petits espaces, et en général dans la mesure des besoins ordinaires de la famille, comme aussi de la somme de maind'oeuvre que ses membres sont en état de fournir.

C'est une exploitation qui est nécessairement à faibles moyens. Par exemple, le battage des grains est bien, pour la plus grande partie, exécuté au moyen de la batteuse mécanique. D'autre part, il ne se fait pas d'un coup et avec l'aide d'une nombreuse équipe d'engagés ou de voisins. Clovis s'y prend à plusieurs fois. Cela lui permet, m'explique-t-il de se charger de toute la besogne, en s'aidant seulement de son fils-associé. Autre avantage : il a toujours 
sous la main, en s'y prenant de cette manière, une quantité de provende à l'état frais.

Les machines agricoles en usage à Saint-Irénée se rattachent presque toutes, - on n'en sera pas surpris, - à la récolte des céréales. Nous avons vu que de bonne heure la batteuse mécanique y avait été introduite. De même, nombre de moissonneuses simples sont utilisées. Cependant, encore en 1929, les moissonneuses-lieuses étaient une nouveauté et une rareté. Elles étaient généralement entre les mains de jeunes gens, fils de cultivateurs, qui faisaient à tour de rôle la visite des diverses exploitations rurales réquisitionnant leurs services.

\section{Le double appui de la culture paysanne : simple récolte des productions spontanées, industries domestiques}

$\underline{\text { Retour à la table des matières }}$

Cette culture familiale combinée avec un élevage restreint ne saurait absorber toute l'activité des membres du groupe, ni parer pleinement à leurs besoins. Voyons comment le cultivateur de Saint-Irénée cherche à combler cette double lacune.

Nous avons vu que, dans ses opérations de culture, l'habitant s'appuie d'une part sur la communauté familiale et, d'autre part, sur la productivité naturelle et les réserves accumulées de fertilité du soi. Voyons quelles autres ressources il va tirer de la libéralité de la grande nature.

Chez Méderile Gauthier et chez Clovis Gauthier, cultivateurs sérieux installés sur le plateau de Saint-Irénée, je ne vois guère que le bois qu'on puisse ranger parmi les productions spontanées dignes de mention. Déjà la forêt, décimée par des années de «pillage » c'est-à-dire d'exploitation désordonnée par les premiers habitants, ne fournissait plus que du bois propre seulement au chauffage, lorsque deux industries nouvelles, permettant d'utiliser même des arbres de petite taille pour la production de la pâte de bois et des bobines, vinrent donner un regain d'activité aux travaux de l'exploitation forestière, en dépit des désastres causés de temps à autre par le feu. 
Il fut un temps où la fourrure fournissait des ressources appréciables aux colons. Même du vivant d'Isidore Gauthier père, il y a soixante-dix ans, la fourrure, dont le rôle avait été si grand dans l'évolution sociale de la colonie, ne comptait que fort peu dans les moyens d'existence de l'habitant. À SaintIrénée, pourtant, on parle toujours de la rencontre que fit ce vaillant Isidore avec une mère ourse, laquelle avait posé sur son épaule une formidable patte. Dans cette circonstance, à ce que rapporte la tradition, le vigoureux colon aurait fait preuve d'un admirable sang-froid en criant à son compagnon, au moment où celui-ci allait décharger son fusil dans le corps de la bête : «Ne tire pas, tu vas gâter sa peau ; donne-lui plutôt de la hache sur la tête ».

Des productions spontanées de la forêt, passons aux productions spontanées de la mer. Au temps d'Isidore Gauthier père, il y a près de trois quarts de siècle, les cultivateurs de Saint-Irénée, riverains du fleuve, exploitaient ce qu'on désigne des « pêches » de fascines, parcs à poissons en claies qui s'avancent au large et se terminent en impasse. Mais les poissons ne fréquentent plus autant ces parages ; cette manne de la mer a beaucoup diminué ; on ne voit presque plus de ces parcs sur la grève de Saint-Irénée. Nous causons quelques instants avec joseph Bouchard, un des derniers à posséder une "pêche », qu'il visite à marée basse. Il n'en exploite pas moins directement sa terre, couchée entre la mer et le plateau.

Le marsouin, qu'on voit parfois s'ébattre aux abords du quai de SaintIrénée, est à l'occasion l'objet d'une chasse qui peut être profitable. Chaque prise rapporte une centaine de piastres, mais la capture de ce petit cétacé n'est pas toujours facile et en outre est fort aléatoire, dangereuse même. Aussitôt qu'il a été atteint, le marsouin disparaît sous l'eau et il est très difficile de le rejoindre. Nombre de jeunes gens ont perdu la vie en se livrant à cette chasse.

\section{E. La disparition graduelle des productions spontanées}

$\underline{\text { Retour à la table des matières }}$

Dans une humble maisonnette, pauvrement garnie, sur la plage même de Saint-Irénée, j'ai pu causer assez longuement avec un type fort original, qui m'a paru représenter de manière frappante la réaction qu'exercent sur une vie humaine la mer et la simple récolte de ses productions spontanées. Patrice Tremblay, natif de l'île aux Coudres, en plein Saint-Laurent, avait, très jeune encore, perdu son père qui s'était noyé, avec plusieurs parents ou amis, en remontant le fleuve dans une goélette à destination de Québec. 
L'orphelin fut adopté par son oncle Louis Tremblay, forgeron à SaintIrénée. Après avoir fait chez cet oncle l'apprentissage du métier, Patrice, âgé de vingt-trois ans, épousa Hortense Gauthier, deuxième fille de notre Isidore, laquelle avait alors dix-huit ans. Sous l'influence, sans doute, de son entourage de terriens et de paysans, le jeune ménage s'en fut s'établir sur le plateau, et, au cours de nos allées et venues dans le rang de Saint-Pierre, entre chez Méderile et chez Clovis Gauthier, on m'a signalé l'endroit où se trouvait naguère la forge de Patrice Tremblay.

Or, loin de la mer, de « cette misérable-là », - pour dire le vrai, Patrice se servait d'un terme beaucoup plus énergique, -cet enfant de l'île aux Coudres s'ennuyait à mourir. Ce fut au point qu'il finit par quitter le plateau, par redescendre sur la plage, où nous l'avons trouvé installé dans une hutte, mais heureux comme un roi. De nouveau en contemplation devant la nappe liquide et son éternelle agitation, le vieux pêcheur s'est senti revivre; il pouvait seiner, pêcher à cœur joie l'éperlan, le capelan et la sardine.

Mais ce vieillard de quatre-vingt-sept ans, fort bien conservé, a ses petits travers. Persuadé qu'en bonne compagnie, ses propos seront charitablement interprétés, il fait parade de ses exploits avec la dive bouteille. Mais il se hâte d'ajouter : «Sans jamais me déranger, par exemple. ». Et sa troisième femme, Adèle Lévesque, de Rimouski, presque aussi âgée que Patrice, renchérit sur la vantardise de son cher époux, et, dans le même esprit, interjette : «je vous dis qu'il en a du vice dans le corps !», suivi de ce commentaire sauveur : « On ne s'ennuie jamais en sa compagnie : toute la veillée se passe à rire, à l'entendre raconter des histoires. »

Or, voici que les productions spontanées sont en train de disparaître. Leur récolte, qui a longtemps soutenu l'imprévoyant, ne saurait plus le nourrir. La pêche de l'anguille, jadis si productive dans ces parages, ne rapporte plus guère maintenant, non plus que la chasse. Il faut se contenter de petites espèces et de prises de mince valeur. Le bois marchand est de plus en plus rare et impose pour sa récolte et son écoulement des frais de plus en plus lourds. L'habitant doit chercher ailleurs un complément de ressources.

Nous avons vu, il y a un instant, deux chefs de famille, entre les plus intelligents du plateau irénéen, chercher la solution du problème dans une culture plus intensive du sol. Méderile Gauthier me confiait qu'il pourrait vendre ses terres, mais qu'il préférait s'agripper à la culture et coopérer à l'établissement de ses fils autour de lui, comme il avait déjà commencé à le faire en 1920. Chez les voisins de Clovis Gauthier, on s'appuie davantage sur les industries domestiques. 


\section{F. La renaissance des arts domestiques}

$\underline{\text { Retour à la table des matières }}$

Une courte promenade dans le rang de Saint-Nicolas, qui prolonge celui de Saint-Pierre, et nous arrivons chez Joseph Gauthier, où nous trouvons la mère de famille et Plusieurs jeunes filles actionnant des métiers doubles. Naguère on pratiquait ces industries en vue seulement des besoins domestiques ou locaux; aujourd'hui, on tisse en vue de la vente, et surtout de la vente au touriste, notamment pour le compte de la «Handicraft Company », des couvertures de lit en coton et fil d'étoupe «boutonné ». Nous échangeons quelques propos avec le grand-père François Gauthier, vénérable octogénaire, qui a été témoin, me dit-il, d'incroyables changements dans son existence, lui qui a vu bâtir, il y a au moins quatre-vingts ans, la maison un peu vieillotte où nous jasons.

Le lendemain, revenus vers l'extrémité nord-est de la paroisse, dans ce rang de Terrebonne où Isidore Gauthier avait son exploitation, nous entrions chez «Ti-Boise » Gauthier, fils d'Ambroise. Nous y trouvions le filage et le tissage de la laine combinés avec la confection de hardes de travail qui avait toujours été en honneur, mais qui est aujourd'hui restreinte aux seuls besoins de la famille. C'est la spécialité de la mère, femme très entendue, l'esprit dirigeant de la maisonnée, à ce qu'il me paraissait bien, comme, du reste, on l'observe fréquemment chez l'habitant.

De leur côté, les hommes, outre la culture de la terre et le soin du troupeau, s'adonnent à quelques industries domestiques, comme la confection de « souliers de beu », mais plus communément à des travaux de terrassement et de réparation sur la voie publique. Le désir de se rapprocher de la grande route, plutôt que la préoccupation d'attirer chez lui le trafic touristique, est probablement ce qui a engagé Ti-Boise, il y a déjà plusieurs années à transporter sa maison d'habitation du bord de la falaise, d'où se découvrait le panorama grandiose du fleuve, à ce bord de route poudreux où défilent les automobiles à l'aller ou au retour de la station balnéaire de la Malbaie.

Il nous a été donné de prendre à la table de Mme Ambroise Gauthier une excellente soupe à la gourgane, suivie d'une omelette encore fumante, avec pain et beurre, thé, confiture et gâteaux; le tout dans de la vaisselle luisante de propreté et sur une nappe de toile de lin de fabrication domestique. Quel touriste songerait à se plaindre de pareil menu campagnard ! 


\section{G. L'habitant dans les mines et les transports}

$\underline{\text { Retour à la table des matières }}$

À diverses époques, il s'est trouvé des cultivateurs de Saint-Irénée pour tenter la fortune autrement que dans la culture du sol. Mais ils n'y ont guère remporté de succès. Vers 1862, voyons-nous dans la monographie de Gauldrée-Boilleau, plusieurs habitants du bord du fleuve s'étaient associés en vue d'exploiter à frais communs des filons métalliques, fer, zinc, plomb, dans les montagnes de l'arrière-pays. Mais faute de capitaux, d'expérience, d'entente entre les associés, ces tentatives échouèrent promptement.

La construction de goélettes, de bricks même, ainsi que leur utilisation pour les transports sur le fleuve, ont été pratiquées de date assez ancienne à Saint-Irénée. Un des frères d'Isidore Gauthier, lisons-nous dans GauldréeBoilleau, «avait eu l'idée de faire construire une goélette d'environ 60 tonneaux. Comme il manquait d'argent pour solder les fournitures effectuées par plusieurs marchands, il était menacé de voir le bâtiment saisi par autorité de justice. Dans sa détresse, il eut recours à Isidore, qui consentit à lui servir de caution pour une somme de 3,000 francs. Par suite d'arrangements subséquents, Isidore acquitta le montant des dettes contractées par son frère, à condition de partager avec lui la propriété du navire. C'est à cette époque qu'Isidore entreprit le service des transports sur le Saint-Laurent. » « Mais, ajoute aussitôt Gauldrée-Boilleau, il ne le continua que pendant deux ans ; il vint à s'apercevoir, en effet, que ses absences nuisaient à la culture de ses terres; il s'empressa de revendre sa part de propriété dans la goélette et acheta une nouvelle terre dans le township de Settrington, à 3 lieues de SaintIrénée. »

Louis Tremblay, forgeron, oncle de notre jovial conteur Patrice, et qui exploitait une forge et une terre à proximité de chez Isidore, s'était, à l'exemple de quelques autres, engagé dans cette petite industrie des transports par voiliers sur le Saint-Laurent; et non pas simplement comme Isidore, dans le dessein de secourir un frère dans l'embarras, mais en vue bel et bien de s'assurer des bénéfices. Or, Louis Tremblay non plus ne put se maintenir longtemps dans l'industrie des transports. Les capitaines qu'il fallait engager pour la conduite de ces petites embarcations faisaient danser trop allègrement les écus de leurs bailleurs de fonds. Nos armateurs d'occasion, après avoir perdu beaucoup d'argent, durent renoncer à leurs entreprises de navigation. 
$\mathrm{Au}$ reste, une survenance notable, une révolution économique, échappant beaucoup plus au contrôle des propriétaires de voiliers que les simples voleries de leurs équipages, allait incessamment couper court à leurs visées ambitieuses : je veux dire le développement des transports à vapeur à la fois par terre et par eau. À Saint-Irénée même, on a la vive sensation du déclenchement de la révolution économique et sociale opéré par l'avènement de cette force nouvelle, à la vue de l'imposante installation dans ce milieu de petites gens d'un millionnaire de Montréal, la grande ville fille des transports, millionnaire dont la fortune, issue du commerce bancaire, s'est déployée ultérieurement dans les entreprises de navigation du Saint-Laurent et d'un chemin de fer dans la région québécoise.

Or l'habitant de Saint-Irénée n'avait pas attendu l'avènement dans sa région des transports à la vapeur pour s'engager dans des entreprises de colonisation à de grandes distances de chez lui. Depuis assez longtemps, des colons agricoles franchissaient en traîneau sur les chemins d'hiver, ou en goélette, durant la belle saison, les 100 milles ou davantage qui, dans la direction du nord, les séparaient des alluvions fertiles bordant le cours mitoyen du Saguenay et les bords du lac Saint-Jean. Un frère d'Isidore, «Dule », ou Théodule, avait depuis nombre d'années gagné la région de Chicoutimi, quand, vers 1867, Isidore partit à son tour. Encore dans la force de l'âge, il n'hésita pas à se défaire de son domaine de la rive fluviale, en plein rapport. Il le vendit à un de ses voisins de Saint-Irénée, ce Louis Tremblay dont il a été question précédemment, forgeron, constructeur, armateur de goélettes, oncle et père adoptif du jovial Patrice, et qui, à ce moment en pleine veine de prospérité, faisait l'acquisition de terres à droite et à gauche. Mieux avisé, notre Isidore, une fois sa terre vendue, s'en allait s'installer à la rivière du Moulin, sur une propriété achetée des grands exploitants forestiers, les Price, tout à côté du centre naissant de Chicoutimi.

À cette phase primitive de la colonisation du Saguenay, c'était, on le conçoit, un long, pénible, voire même périlleux voyage que la traversée en traîneau de la solitude neigeuse qui reliait la région québécoise du bas SaintLaurent à celle du fjord saguenayen. Une des filles d'Isidore Gauthier dont nous parla Gauldrée-Boilleau, Démerise, que nous eûmes la bonne fortune, ma femme et moi, de retrouver à Chicoutimi, en 1929, veuve octogénaire de François Pilote, nous parut être la digne représentante de cet âge héroïque du défricheur canadien.

De taille élevée, à solide charpente, à la voix masculine, apparemment en parfaite santé malgré son grand âge, Mme Pilote nous fit le récit pathétique d'une de ces odyssées qu'elle entreprit, jeune femme, à travers cette projection du plateau laurentien, en compagnie de plusieurs membres de sa famille, pour aboutir à la rivière du Moulin où était situé le lot concédé à son père. Dans ce 
coin de pays perdu, accidenté, le colon n'avait pas à sa disposition les belles routes nivelées, empierrées, dont depuis on a gratifié à grands frais touristes et automobilistes. Le chemin, à peine tracé, suivait au petit bonheur les sinuosités du vallon, les anfractuosités du plateau rocheux ; et sous la conduite d'un charretier malhabile, ou rendu téméraire par de trop fréquentes libations, le voyageur courait le risque de verser dans un banc de neige, sinon de rouler au fond d'un précipice. Puis, le soir, en guise d'hôtellerie on n'avait pour tout abri que la hutte en troncs d'arbres de quelque trappeur ou bûcheron.

Pourtant, c'était là, semble-t-il, un sport délectable, une vraie partie de plaisir pour cette génération robuste, élevée à la dure, et que n'avaient pas amollie les raffinements du confort moderne. Aussi bien, elle était loin d'être luxueuse, elle n'était même pas bien close, à l'abri des intempéries, loin de là, la première maison où Isidore Gauthier, père, dut se loger avec sa famille, en 1867, sur la terre achetée des Price ; et leur seconde habitation ne valait guère mieux; construction hâtivement érigée pour servir de chapelle temporaire aux premiers colons; les Gauthier en ont gardé longtemps le douloureux souvenir.

Mais quel merveilleux progrès depuis soixante ans ! Quelle transformation frappante s'est opérée ! Et d'abord, pendant une douzaine d'années, l'exploitation bénéficia de la vigoureuse emprise d'Isidore père, qui amorça le défrichement de la forêt et construisit cette spacieuse maison, laquelle, du haut de sa colline, domine superbement le lit étroit de la rivière du Moulin et le cours plus large de la sauvage Saguenay. Les quinze ou seize années suivantes, la terre resta aux mains plus débiles et plus vacillantes, à ce qu'il semble, d'Isidore fils, mort en 1894, âgé seulement de quarante-trois ans.

Ensuite, la terre fut quelques années sous la direction de la veuve d'Isidore fils, femme instruite, entendue, dans sa jeunesse institutrice à Jonquière. Elle a convolé en secondes noces avec Hippolyte Tremblay, dit Pierriche, originaire de Saint-Irénée, mais qui a su s'amasser du bien dans les durs travaux de l'exploitation forestière, à l'Anse Saint-Jean, sise à mi-chemin entre Chicoutimi et le confluent de la Saguenay avec le Saint-Laurent. Lors de mon passage à Chicoutimi, en 1929, pendant que, dans un salon garni avec goût, je cause avec la veuve d'Isidore Gauthier fils, devenue Mme Tremblay, son mari valétudinaire, alité dans une chambre voisine, requiert sa présence; et je puis me rendre compte de quels soins et de quelles attentions il est l'objet dans cette maison hospitalière.

Et maintenant, depuis nombre d'années déjà la terre est exploitée, avec un regain d'énergie et de savoir-faire, par Isidore Gauthier petit-fils. Il a arrondi le domaine de 120 arpents que lui avait légué son père et l'a doté de bâtiments spacieux de belle apparence, et pourvus de toutes les améliorations modernes : fond bétonné, eau courante, éclairage à l'électricité, stalles en fer. À part trois chevaux de travail, notre cultivateur avancé ne garde que des bêtes de choix : 
vaches de race Ayrshire pure, inspectées, au nombre de trente ou trente-cinq; porcs de race Yorkshire. Le propriétaire me fait faire le tour de ses champs ; ils portent en ce moment de belles récoltes de céréales, qu'on est précisément à moissonner. Il me signale les diverses natures de sol : sableux sur les crêtes, argileux à mi-côte, tourbeux dans les fonds. La meilleure terre arable est la franche, où l'argile se

plus ou moins de sable ou de terre végétale. Les sols bas ne sont pas productifs à moins d'avoir été drainés au préalable; et les colons qui ont négligé de drainer à fond les terres humides sont les seuls à se plaindre du climat de la région.

Mais ce qui chez Isidore III (ou petit-fils) m'a frappé encore plus que la bonne installation matérielle du foyer, que la bonne tenue de l'exploitation, que toutes ces manifestations de progrès économique qui partout nous apparaissaient, c'est la fécondité remarquable de cette famille de cultivateurs prospères et les promesses d'avenir que donnait cette remuante jeunesse. Ces deux époux qui n'avaient pas atteint la cinquantaine comptaient déjà treize enfants, dont dix survivants. L'aîné, le seul qui eût manifesté du goût pour les carrières libérales, s'était, après une année de théologie au collège de Chicoutimi, tourné vers la médecine, qu'il étudiait depuis quatre ans à l'université de Québec. Le second fils secondait son père dans l'exploitation du domaine, tandis que le troisième s'était spécialisé dans l'élevage du renard pour le compte d'une compagnie. Une grande fille, Marie des Neiges, prêtait main-forte à sa mère dans la tenue de la maison. Les autres, garçons ou filles, se préparaient vaillamment à gagner leur vie.

\section{H. Fonction éducatrice de la famille}

\section{Retour à la table des matières}

En vue de compléter et de mener à bien cette étude monographique de la famille rurale canadienne, nous allons retourner à Saint-Irénée. Déjà nous avons observé sous plusieurs de ses aspects ce groupement initial. Nous l'avons considéré d'abord comme simple agrégat de parents installés au même foyer; puis, comme réunion de travailleurs coopérant dans l'ordre économique : culture, simple récolte, fabrication, transports, et par surcroît engagée dans l'œuvre de la colonisation. Mais à cela ne s'arrête pas le rôle social de la famille canadienne : elle exerce en outre une fonction administrative, puisque dans le cours ordinaire des choses elle accumule des biens, dont elle fait un emploi déterminé et qu'elle transmet en tout ou en partie à ceux de la 
génération suivante. Héritage de double nature : biens matériels, d'abord, sous forme de terres, de bâtiments, de troupeaux, d'instruments de travail, etc. ; et aussi, héritage de biens spirituels, comme diraient les sociologues philosophes, sous forme de traditions, de coutumes, de règles de conduite. De ce fait, la famille est investie forcément d'une mission éducatrice ; et même, c'est sa fonction éducatrice et la manière dont elle la remplit qui permettent le mieux de la caractériser et de la classer exactement.

Nous l'avons vu dans les premières pages de la présente étude, la monographie du Paysan de Saint-Irénée, écrite en collaboration, semble-t-il, par Gauldrée-Boilleau et son compatriote le curé Mailley, assimile le groupement familial des Gauthier au type de la famille souche, si chère à LePlay. Or l'analogie est loin d'être parfaite : par suite à la fois des conditions géographiques et des circonstances historiques de son développement, notre famille rurale est beaucoup moins traditionnelle, beaucoup moins rivée en un point du territoire, beaucoup moins communautaire à certains égards, que les familles de montagnards pyrénéens, par exemple, distinguées par LePlay sous l'étiquette de la famille souche. Cinq ou six ans ne s'étaient pas écoulés depuis la visite de Gauldrée-Boilleau et l'encre n'était pas encore sèche sur cet éloge dithyrambique de la famille souche canadienne, que le fameux « centre traditionnel » des Gauthier de Saint-Irénée était délaissé, la maison « débâtie », et les membres du groupement familial dispersés, ou transplantés au loin.

La famille de l'habitant ne doit pas davantage être confondue avec un autre type que LePlay a placé dans cette même catégorie de la famille souche : je veux dire la famille particulariste de certaines populations du nord de l'Europe. L'habitant ne forme pas ses enfants à l'initiative particulière dans la mesure où cela se fait en milieu particulariste; il reste plus ou moins empêtré dans ses habitudes de dépendance communautaire; il néglige trop l'instruction, surtout celle de l'ordre élémentaire pratique, qui lui serait d'un indispensable secours pour s'élever dans l'ordre social. De ce groupement de robustes travailleurs manuels, il ne sort guère, sauf de manière exceptionnelle, accidentelle, transitoire, une élite de chefs d'entreprise dans les arts usuels. Une fois que le domaine a acquis les dimensions suffisantes pour parer aux besoins ordinaires de la famille et subventionner dans une mesure variable l'établissement des jeunes, il reste stationnaire ou se subdivise. La race se décapite à chaque génération. 


\section{La double réaction du type familial sur la société canadienne}

$\underline{\text { Retour à la table des matières }}$

La mise en oeuvre a la base de la société canadienne d'un groupement familial à fonction aussi compréhensive, à capacités aussi diverses, devrait être pour nous, semble-t-il, un gage de supériorité, et à certains égards, cela est incontestable. Ce qu'on désigne parfois de miracle canadien, le miracle de la survivance du Canada français, a là son explication : l'habitant se suffit à lui-même.

Mais si l'on y regarde de plus près, on s'apercevra que ce trait élémentaire de notre économie sociale a d'autres répercussions de caractère beaucoup moins favorable. Cela apparaîtra nettement, je pense, si, déjà initiés comme nous le sommes au fonctionnement interne de la famille rurale, nous l'observons dans ses rapports avec les autres groupements connexes ou superposés.

Représentez-vous une simple juxtaposition de familles semblables à celles que nous venons d'observer : à peu près toutes engagées dans la culture du sol et s'y maintenant chacune par le travail en commun de ses membres, ne recourant que dans des cas exceptionnels à la main-d'œuvre salariée ; complétant les ressources extraites du sol par la simple récolte de productions spontanées de la terre ou des eaux, ou par l'exercice de diverses industries domestiques ou de divers métiers accessoires. Ces familles seront toutes égales ou du moins, elles ne seront pas hiérarchisées. Ce que chaque famille ne saurait exécuter ou produire par l'effort concerté de ses membres, elle se l'assurera autant que possible avec le concours bénévole de ses voisins, à charge de revanche. C'est la constatation faite par Gauldrée-Boilleau en 1861; et l'état de choses n'a pas très sensiblement varié depuis.

À Saint-Irénée, de nos jours, il n'existe guère plus qu'au moment où Gauldrée-Boilleau et le curé Mailley rédigeaient leur monographie, une classe patronale. Comme il le faisait alors, comme effectivement il l'a fait depuis la fondation de la colonie française, l'habitant se patronne lui-même, mais sans, de son côté, patronner personne autre que ses enfants. Aussi la division du travail ne se produit-elle pas pour la peine : les professions auxiliaires, faute de clientèle, ne sauraient se développer. Le commerce local et les arts libéraux végètent. 


\section{J. Action du commerce lointaine et pourtant dissolvante}

$\underline{\text { Retour à la table des matières }}$

Une page curieuse de la monographie de Gauldrée-Boilleau donne bien l'idée de la faible activité du commerce local à Saint-Irénée, il y a quelque soixante ans. L'argent est rare, dit-il ; les habitants sont obligés de recourir au mode primitif du troc, de l'échange en nature, soit entre eux, soit avec les étrangers. Les petits marchands acceptent en paiement de leurs fournitures en épiceries, étoffes, quincailleries, etc., des céréales et es volailles. Les créanciers pour des sommes plus importantes se mettent à construire des goélettes de 30 à 40 tonneaux, et réquisitionnent leurs débiteurs pour des pièces de bois ou des journées de travail.

Je signalais, il y a un instant, l'imposante installation d'un capitaliste enrichi dans la finance et les transports. Mais, bien entendu, ce courtier montréalais n'a pas bâti sa fortune sur place. Saint-Irénée n'a été pour lui qu'une retraite champêtre, un lieu de repos et une occasion de dépense. Greffé tardivement sur ce milieu rural qui n'était pas le sien, mais qu'il a fait largement profiter de ses dons et même représenté quelque temps au Parlement, M.F... a vécu, peut-on dire, en marge de ce groupement communautaire et paroissial dont il n'était pas un produit.

D'autre part, pour n'être pas entré de plain-pied dans le mouvement mondial des transports et des échanges, Saint-Irénée n'en a pas moins ressenti de bonne heure certains effets pernicieux résultant de la brusque apparition du commerce dans un milieu insuffisamment prémuni contre ses tentations. Dès 1861, au témoignage de l'auteur de la monographie, la prodigalité, le luxe exercent leurs ravages dans cette campagne reculée :

«Il y a quelques années, on ne voyait aux hommes et aux femmes que des vêtements fabriqués à la maison, avec la laine de leurs brebis ; aujourd'hui, on ne s'en contente plus : le luxe fait des progrès parmi les habitants; les étoffes des manufactures anglaises, aux dessins variés et aux couleurs éclatantes, excitent toutes leurs convoitises. L'usage des corsets et de la crinoline commence même à se répandre. »

À la messe à Saint-Irénée, le 5 septembre 1920, sur la falaise dominant le fleuve, dans une église d'apparence antique dont l'architecture remontait bien à 
trois quarts de siècle, sinon à tout un siècle en arrière, dans la foule des fidèles, je n'ai pu discerner un seul homme, une seule femme, portant des étoffes du pays. Les robes de soie et les tissus aux couleurs voyantes étaient très en évidence.

On le voit, dans cette paroisse rurale isolée, la famille de l'habitant, avec sa formation quasi-communautaire, repliée sur elle-même, qui la dresse à parer directement à tous ses besoins matériels, et dès lors restreint au possible la division du travail social, offre bien peu de prise aux opérations du commerce. Pourtant, dès les premières atteintes de cette force nouvelle qui s'exerce du dehors, une notable partie de la population se trouve ébranlée, instabilisée. C'est là le signe d'une évidente faiblesse, d'une insuffisance notoire de l'organisation de la vie privée du Canadien. Mais poursuivons notre analyse.

\section{K. Rôle effacé de la culture intellectuelle et des Professions libérales}

$\underline{\text { Retour à la table des matières }}$

Voici bien une autre faiblesse tout aussi grave, sinon plus grave, que la première : cette famille traditionnelle de l'habitant est peu portée vers l'instruction. En 1861, comme le constate l'auteur de la monographie, « l'éducation n'est pas aussi avancée à Saint-Irénée qu'on serait en droit de le souhaiter. Il n'y a guère que les adolescents qui sachent lire et écrire. Les habitants parlent assez correctement la langue française. Aucun d'eux ne comprend l'anglais, hormis quelques pêcheurs qui l'entendent à peu près, sans être en état de s'exprimer que d'une manière très imparfaite ».

Notamment, Isidore Gauthier, un des hommes les plus remarquables et un des esprits dirigeants de la paroisse, n'a reçu aucune instruction; il sait seulement un peu lire. Au temps où il était enfant, il n'y avait pas encore d'école sur place, la loi qui inaugurait les écoles publiques n'ayant été adoptée par l'assemblée législative qu'en 1846. En 1861, Saint-Irénée est bien doté de deux écoles élémentaires et d'une école modèle, qui date de l'année précédente. Ce sont des filles de cultivateurs, non mariées, qui y enseignent sous la surveillance d'une commission scolaire dont le curé est président. Il ne se manifeste pas moins à l'occasion, un fort sentiment de méfiance pour les « petites écoles ». «L'habitant de Saint-Irénée, dit encore Gauldrée-Boilleau, ne comprend pas encore tout à fait l'importance de l'instruction. Aussi voit-on 
quelquefois, à l'époque fixée pour la rentrée des cotisations, les commissaires des écoles en lutte ouverte avec une partie de la population. » Et cela en dépit des efforts du curé qui les rabroue d'importance.

Observez que cet habitant, à l'origine du moins, n'est guère mieux disposé à l'égard de l'instruction supérieure. Mais c'est pour des motifs différents et qui paraissent en somme justifiés, de l'aveu même de l'auteur de la monographie. Si l'habitant de prime abord se montre indifférent, hostile même au mouvement scolaire, qui pourtant lui procurerait un commencement d'instruction et le mettrait en mesure de s'élever quelque peu dans l'échelle sociale, c'est qu'il n'a pas d'ambition de ce côté et ne voit dans la culture du sol qu'une routine manuelle. À ses yeux, la loi des écoles et la commission scolaire ne sont pour les gouvernants qu'un prétexte à le taxer, tout en le privant du secours de ses enfants dans l'exploitation de la ferme. Quant aux enfants, habitués dès leur jeune âge à la vie au grand air et à un constant exercice de leur activité physique, sans le contrepoids de la culture intellectuelle, ils n'ont Pas, sauf exception, le goût de l'étude et répugnent à la réclusion, même passagère, de la «petite école ».

Mais comment expliquer la froideur de l'habitant pour l'enseignement secondaire des couvents, des collèges et pour l'enseignement supérieur de l'université, qu'aucune loi ne le contraint à subventionner et où il est à même de faire instruire, sinon héberger, ses enfants à des prix très modiques ? Dit Gauldrée-Boilleau : «Bien que plusieurs habitants de Saint-Irénée disposent de moyens pécuniaires qui leur permettraient d'envoyer leurs enfants dans les collèges, ils n'ont pas cette ambition, et s'appliquent uniquement à en faire des cultivateurs laborieux. »

Cette apathie semble s'expliquer assez bien par des raisons de trois ordres, agissant isolément ou en combinaison. Il y a d'abord la raison d'ordre économique. L'habitant, de type quasi-communautaire, tend à tous égards à se suffire directement à lui-même ; il se montre réfractaire à la division du travail social en matière intellectuelle comme en matière pratique. En deuxième lieu, il y a une raison d'ordre historique : il reste empêtré dans la tradition sous la forme du folklore médiéval ou même antique. Un exemple frappant nous en est fourni Par la monographie du Paysan de Saint-Irénée.

« Il n'y a point de vétérinaire à Saint-Irénée. Quand les chevaux ou les bestiaux tombent malades, on s'adresse à quelque empirique qui s'est acquis une réputation par ses cures merveilleuses. Notre homme demande à rester seul dans l'étable avec l'animal confié à ses soins; il se découvre, fait le signe de la croix et, les yeux levés au ciel, il récite une courte prière en l'honneur de saint Pierre. Il sort ensuite et affirme, avec un sang-froid imperturbable, que l'opération est achevée, que la bête guérira et qu'il n'y a d'autre soin à prendre que de lui administrer un remède fort simple qui consiste généralement en lait chaud, saupoudré de poivre et de sucre. » 
L'influence débilitante de la tradition quasi-communautaire se manifeste sous un autre aspect: dans la faible valeur morale d'un grand nombre de ces fils d'habitants, établis à la ville ou à la campagne, et cela même après qu'ils sont passés par la forte discipline de l'internat, ou « collège classique », où des ecclésiastiques enseignent les littératures, la philosophie scolastique et un peu de sciences.

«Les paroissiens de Saint-Irénée, écrit encore Gauldrée-Boilleau (avec le concours, sinon sous l'inspiration directe du curé de la paroisse), prétendent que la plupart des enfants que l'on envoie dans les collèges de Québec y perdent la foi religieuse et souvent y contractent de mauvaises habitudes. Ils tirent leurs preuves de la conduite des «seigneurs », médecins, notaires et avocats du comté. À les en croire, cette classe de gens lettrés prêterait assez fréquemment au scandale par une conduite peu mesurée, des propos irréligieux et l'absence de scrupules dans les transactions monétaires. Ces accusations dérivent en partie des sentiments de jalousie qui, dans les campagnes, existent à peu près partout contre les classes bourgeoises ; mais il faut reconnaître que les mœurs des personnes indépendantes de fortune ou exerçant des professions libérales contrastent jusqu'à un certains point au Canada avec le caractère simple et les pieuses croyances des cultivateurs. »

Un dernier motif de défiance que nourrit l'habitant de Saint-Irénée du milieu du siècle dernier à l'encontre de l'enseignement des collèges est d'ordre tout pratique. C'est que les professions libérales auxquelles cet enseignement prépare, subsidiairement à l'état ecclésiastique, se sont trouvées promptement encombrées dans ce milieu où la richesse, produit de l'industrie et du commerce, ne se développait, on le conçoit, que lentement et sporadiquement. Aussi l'auteur de la monographie loue-t-il les habitants du «bon sens » dont en cela ils font preuve. «Effectivement, on ne compte dans les villes que trop de médecins, d'avocats, de notaires. Ce qu'il faut au Bas-Canada, c'est avant tout des agriculteurs. » Ainsi le même vice de formation sociale qui faisait du commerce un élément d'instabilité plutôt que de bien-être pour la société canadienne fait pour elle de l'instruction presque un meuble inutile, sinon un moyen de déracinement et de déclassement d'une notable partie de sa classe rurale.

Lors d'un bref séjour à Saint-Irénée en 1920, comme au cours d'une visite un peu plus prolongée au même endroit en 1929, je n'ai rien observé indiquant un changement notable d'attitude de la population locale en ce qui regarde le commerce ou l'instruction. Sans doute le folklore, les conceptions populaires surannées ont perdu de leur prise sur les esprits ; des écoles mieux aménagées fonctionnent sous l'égide d'une administration scolaire acceptée de tous. Mais le zèle pour l'instruction n'est pas aussi vif ou général qu'on pourrait le désirer. Une grande école installée sur la plage, par la munificence du richard montréalais dont il a été question, et qui avait été confiée aux soins de religieuses enseignantes, a dû, par suite de dissensions intestines, fermer ses portes.

Encore à ces dates relativement récentes, les professions libérales n'étaient guère représentées que par quelques vieux magistrats de l'extérieur qui 
venaient y passer la belle saison en villégiature. Pas même de médecin sur les lieux. Pour les maladies graves, on faisait venir un homme de l'art de l'un des bourgs voisins, les Éboulements ou la Malbaie. Un vétérinaire qui avait planté sa tente à Saint-Irénée a dû, faute de clientèle, aller chercher fortune ailleurs.

\section{Les deux corporations dirigeantes}

$\underline{\text { Retour à la table des matières }}$

Jusqu'ici, dans notre analyse du milieu social de Saint-Irénée, nous n'avons discerné en dehors de la famille de l'habitant aucun élément directeur., Ni l'industrie ni le commerce ni aucune des professions libérales ne nous l'ont fourni. Le voisinage rural, sous la forme du rang (dont il se trouve cinq ou six à Saint-Irénée) organise bien spontanément certains services d'utilité publique, mais qui sont de nature très simple et intermittente. Comme le déclare nettement Gauldrée-Boilleau, « le principe de l'association n'a pas été compris ni exploité par les Canadiens d'origine française, comme il l'a été par leurs concitoyens d'origine anglaise ».

Or cette inaptitude des Canadiens français à s'associer dans la vie privée, sauf pour les objets les plus essentiels et dans les conditions les plus simples, ils la manifestent également dans le domaine de la vie publique. Aussi, «le gouvernement de la province qui ne s'immisce presque jamais dans les affaires locales, dont il laisse habituellement le contrôle exclusif aux habitants de la paroisse, s'est-il appliqué dernièrement à répandre, parmi la population française du Bas-Canada, l'esprit d'indépendance qui règne à cet égard parmi les populations anglaises du Haut-Canada ».

Cette énergie organisatrice, directrice de la vie collective et publique dont l'habitant n'a pu trouver les éléments à l'intérieur de son groupement familial ou de son voisinage rural, c'est un type d'association à formes plus rigides, c'est la corporation religieuse et c'est la corporation politique qui vont la lui fournir. Fait curieux, caractéristique, l'habitant qui, de date très ancienne, a su trouver dans son groupement familial l'armature d'une vie économique simple, mais intégrale à son point de vue du moins, a reçu de l'extérieur, et pour ainsi

dire toutes faites, ses institutions religieuses, qu'il tient de l'Église catholique, et ses institutions politiques, empruntées à l'Angleterre, et, à un moindre degré, aux États-Unis.

La religion catholique est pratiquée avec ferveur par les habitants de SaintIrénée ; les communions sont nombreuses à toutes les fêtes marquantes; la 
prière du soir se dit en commun dans la plupart des familles ; on fait célébrer des messes pour les défunts, ou en vue d'obtenir des grâces particulières; on verse une contribution annuelle pour la Propagation de la Foi. Il n'y a dans la paroisse qu'un seul individu qui ne reçoive pas les sacrements dans la quinzaine de Pâques, et c'est un usurier qui prête des fonds à 12 p. 100. Dans les campagnes on se réjouit plutôt qu'on ne s'afflige de la mort d'un enfant en bas âge, parce que c'est « un ange acquis au ciel ».

«L'autorité du curé est respectée, il jouit de l'estime que commande celui dont la mission est d'enseigner et de commenter l'évangile. On a la ferme croyance que le prêtre entretient avec le ciel des relations surnaturelles. C'est au ministre du culte que les habitants s'adressent dans toutes les affaires importantes, soit civiles, soit religieuses. Son intervention prévient les procès ou les termine, apaise les haines, réconcilie les ennemis. La loi civile ne les touche pas; ils s'en rendent à peine compte. La religion est au contraire pour eux un frein très puissant, et le seul capable de les retenir. »

Telle était la situation éminente du curé, au témoignage et presque dans les termes mêmes de Gauldrée-Boilleau, qui écrivait vers 1861. Et pourtant, le curé d'alors, arrivé récemment de France avec des idées autoritaires et des allures cassantes, était, au témoignage de contemporains, moins apprécié que d'autres à procédés conciliants.

À cette époque encore le clergé est rétribué d'après l'ancien mode traditionnel, médiéval, de la dîme ; ou, pour plus d'exactitude, le curé a droit au vingt-sixième minot des céréales récoltées par chaque famille d'habitant. «Le clergé est à la tête de l'œuvre de la colonisation. On voit les prêtres explorer eux-mêmes la contrée, choisir et désigner les endroits qui semblent les plus favorables à l'établissement de nouveaux centres de population et prêcher d'exemple en s'y installant eux-mêmes au milieu de privations de plus d'un genre. »

Par l'intermédiaire surtout des évêques, l'influence du clergé catholique est prépondérante au conseil de l'instruction publique de Québec. Il est dans la province comme le défenseur attitré de la langue française et des traditions importées de l'ancienne mère patrie française. Enfin, il joue parfois le rôle d'intermédiaire en quelque sorte entre ses ouailles et les autorités anglaises, auxquelles il a plus d'une fois prêté un utile concours. Et l'auteur de la monographie ajoute en termes caractéristiques : "Les rapports mutuels du clergé et du gouvernement de S. M. britannique sont parfaitement dignes. »

Il n'y a donc rien dans les rapports de l'Église avec les institutions politiques supérieures qui soit de nature a gêner ou restreindre son action religieuse. La seule limitation de son influence ne saurait provenir que de la formation sociale même de ses fidèles. Or cette même tradition communautaire qui au foyer familial entrave l'action religieuse en diminuant la valeur 
personnelle du sujet, est aussi un obstacle au bon fonctionnement de la vie politique.

Durant le siècle et demi que dura la domination française en Amérique, les colons de la Nouvelle-France ne jouirent jamais d'un régime de liberté politique comparable à celui que nous possédons aujourd'hui ; et il s'écoula tout près d'un siècle du présent régime anglais avant que les efforts combinés de dirigeants recrutés dans l'une et l'autre race eussent réussi à nous faire concéder cette autonomie par les fonctionnaires de Downing Street.

$\mathrm{Au}$ moment où Gauldrée-Boilleau recueillait les matériaux de la monographie du Paysan de Saint-Irénée, notre victoire était complète : après la liberté religieuse, nos pères avaient conquis, en bonne partie du moins, l'autonomie politique. Le « gouvernement responsable » était un fait accompli, et déjà se préparait la fédération des provinces canadiennes.

Mais à ce moment où en était rendue l'éducation politique de nos Canadiens ? Gauldrée-Boilleau, en observation à Saint-Irénée, nous laisse voir clairement que, par suite de l'indifférence des uns, de la méfiance ou des mauvaises passions des autres, même de la malhonnêteté de plusieurs, enfin de la corruption électorale, le fonctionnement du régime nouveau laissait fortement à désirer. Ceux qui s'intéressaient le plus vivement à la lutte électorale souvent ne se souciaient que médiocrement de la valeur intrinsèque des mesures débattues. Ils se laissaient emporter par leurs prédispositions personnelles en faveur de tel candidat ou de tel chef de parti.

Même à l'occasion, on n'hésitait pas à recourir à la violence. En temps d'élections, les frères d'Isidore Gauthier, peut-être Isidore lui-même, auraient volontiers fait le coup de poing avec un adversaire politique quelconque. C'était comme un réveil de l'esprit combatif engendré par des habitudes de l'ancien clan celtique. La conduite louche ou malhonnête des gens en place était à tort ou à raison le motif fréquent de ces luttes intestines.

« Nous sommes simples, nous autres habitants », disait un jour Isidore à son curé, « et vu notre ignorance, nous sommes contraints de mettre à la tête de nos municipalités et de nos administrations des citoyens instruits, mais qui, au fond, nous exploitent et ne méritent peut-être pas l'estime des coeurs honnêtes. »

Tous, sans doute, nous sympathisons avec Isidore dans sa détresse; mais il n'y a qu'un remède au mal dont il souffre, lui et les siens et dont nous souffrons tous tant que nous sommes : c'est l'orientation de plus en plus énergique et complète de la famille rurale canadienne-française dans le sens de l'initiative particulariste. Ainsi nous assurerons-nous une élite de chefs véritables dans tous les départements de l'activité sociale. 